\title{
Magnetic Imaging. Excuse me, what is Relevant for High Density Recording Technology?
}

Joachim Ahner, Seagate Research, 1251 Waterfront Place, Pittsburgh, PA 15222-4215

Despite the invaluable contribution of MFM (magnetic force microscope) imaging has made to the development of high-density magnetic storage devices in the past, the increase of magnetic storage density is based on the development of recording media comprised of stable magnetic domains with diameters in the order of $10 \mathrm{~nm}$ and below. Commercial MFM technology (resolution 30 nm) has been "outpaced" by the development of the magnetic storage technology. Seagate has developed HRMFM (high resolution magnetic force microscope) technology and has demonstrated a lateral resolution of about $10 \mathrm{~nm}$, the highest resolution in MFM technology reported so far. However, for transition parameter investigation as cross track correlation length and transition noise, even higher resolution is required. I will present newest developments in magnetic imaging technology at Seagate including relevant image analysis and novel HR-SEMSD technology (high resolution scanning electron microscopy with spin selective detection). 